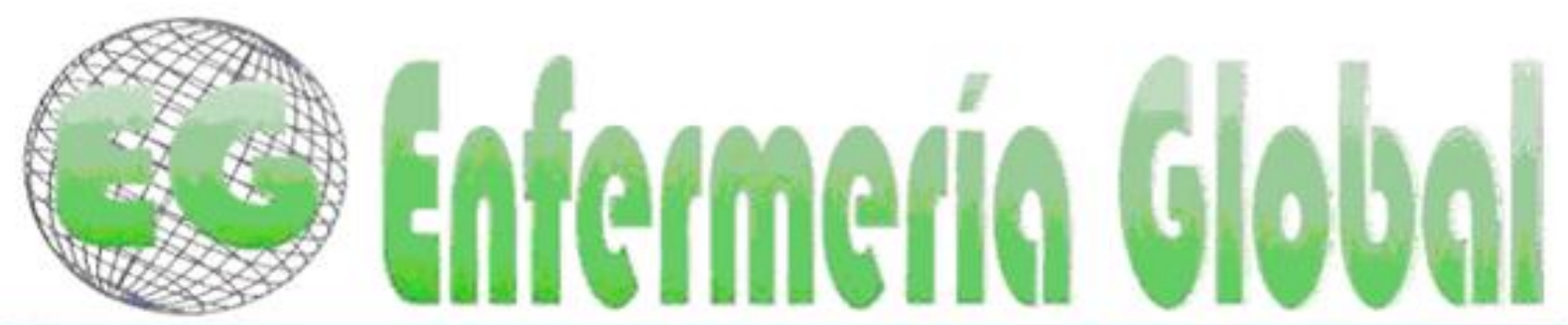

ISSN 1695-6141

Revista electrónica trimestral de Enfermeria

No38

www.um.es/eglobal/

Abril 2015

\title{
CLÍNICA
}

\section{Cateterismo urinario permanente: práctica clínica}

Cateterismo urinário de demora: prática clínica

Urinary catheterization delay: clinical practice

\section{"Mazzo, Alessandra **Bardivia, Carolina Beltreschi ${ }^{* * *}$ Jorge, Beatriz Maria ${ }^{* * *}$ Souza Júnior,Valtuir Duarte ${ }^{* * *}$ Fumincelli, Laís ${ }^{* * *}$ Mendes, Isabel Amélia Costa}

\author{
"Enfermera. Profesor Doctor. "**Graduanda en Enfermería. "***Enfermero. Programa de Posgraduación \\ en Enfermería Fundamental ****Enfermera. Profesor Titular. E-mail: iamendes@usp.br Escuela de \\ Enfermería de Ribeirão Preto, Universidad de São Paulo (EERP-USP). Centro Colaborador de la OMS \\ para el Desarrollo de la Investigación en Enfermería. Brasil
}

\author{
Palabras Clave: Cateterismo Urinario; Enseñanza, Enfermería; Técnicas \\ Palavras-chave: Cateterismo Urinário; Ensino; Enfermagem; Técnicas \\ Keywords: Urinary Catheterization; Teaching; Nursing; Techniques
}

\section{RESUMEN}

Objetivo: Verificar cómo el procedimiento de inserción del catéter urinario permanente ha ocurrido en la práctica clínica de enfermería.

Método: Estudio observacional, exploratorio y descriptivo, desarrollado en hospitales de un municipio del interior del estado de São Paulo, Brasil. Las premisas éticas fueron cumplidas (Parecer 233/2008/Comité de Ética en Investigación de la Escuela de Enfermería de la Universidad de São Paulo). Los datos fueron recolectados mediante entrevista, utilizándose un instrumento semiestructurado. De las trece instituciones del municipio, nueve participaron del estudio.

Resultados: En las instituciones investigadas, el procedimiento es practicado de manera ritualista respecto al método y los materiales.

Conclusión: Para practicar el cateterismo urinario permanente es necesario mayor compromiso de los profesionales y mayores inversiones en investigaciones para esclarecer los aspectos que todavía no pueden ser comprobados. 


\section{RESUMO}

Objetivo: Verificar como o procedimento de inserção do cateter urinário de demora tem se dado na prática clínica da enfermagem.

Método: Estudo observacional, exploratório e descritivo, realizado em hospitais de um município do interior paulista. Seguidos os preceitos éticos (Parecer 233/2008/ Comitê de Ética em Pesquisa da Escola de Enfermagem de Ribeirão Preto da Universidade de São Paulo), os dados foram coletados por entrevista, utilizando-se um instrumento semi-estruturado. Dentre as treze instituições do município, nove participaram do estudo.

Resultados: Nas instituições pesquisadas o procedimento é realizado de forma ritualista quanto ao método e materiais.

Conclusão: Para realização do cateterismo urinário de demora é necessário maior comprometimento dos profissionais e maiores investimentos em pesquisas para esclarecimento dos pontos que ainda não podem ser comprovados.

\section{ABSTRACT}

Objective: Verify how the urinary catheterization delay procedure has taken place in clinical nursing practice.

Method: Observational, exploratory and descriptive study, undertaken at hospitals in an interior city in the State of São Paulo, Brazil. The ethical premises were complied with (Opinion 233/2008 University of São Paulo at Ribeirão Preto College of Nursing Research Ethics Committee). The data were collected through an interview, using a semi structured script. Nine out of 13 institutions in the city participated in the study.

Results: At the participating institutions, the procedure is performed ritualistically in terms of method and materials.

Conclusion: To practice urinary catheterization delay, greater professional commitment and further investments in research are needed to clarify aspects that cannot be proven yet.

\section{INTRODUCCIÓN}

El cateterismo urinario es utilizado en pacientes con dificultades o imposibilidad de orinar. Consiste en la introducción de una sonda hasta la vejiga para retirar la orina $^{(1,2)}$.

La introducción de instrumentos a través de la uretra para retirada de la orina se remonta a épocas antiguas. Los primeros registros de su práctica pueden ser verificados entre los egipcios; en aquella época, eran utilizados tubos huecos de cobre y laca. Posteriormente, griegos, romanos y chinos emplearon instrumentos parecidos. Sin embargo, fue en el siglo $X$ cuando aparecen los registros de las primeras sondas uretrales flexibles, confeccionadas con cuero animal y únicamente en el siglo XIX, debido al procesamiento del caucho y a las técnicas innovadoras de la urología francesa, puede observarse el avance en la confección de los catéteres uretrales $^{(2)}$.

El procedimiento de inserción del catéter uretral debe ser estéril o limpio, según el tiempo de permanencia del catéter. Siempre trae riesgos de trauma e Infección del Tracto Urinario (ITU). 
En el cateterismo urinario de alivio e intermitente, los catéteres son removidos después del vaciado de la vejiga, lo que implica menores tasas de ITU. En el cateterismo urinario permanente, los riesgos para ITU son más significativos. La ITU provocada por el uso del catéter urinario permanente tiene una relación estrecha con el tiempo de permanencia y con el creciente número de pacientes que usan el catéter. Trae repercusión económica, secuelas, complicaciones y grandes daños a la población ${ }^{(2,3)}$.

Los traumas causados por la inserción del catéter urinario muchas veces no son diagnosticados, resultando en lesiones y falsos trayectos, que pueden o no ser acompañados de sangrado e infecciones del tracto urinario; provocan manifestación dolorosa, oriunda de la fricción del catéter mal lubrificado contra la mucosa uretral y/o de las maniobras agresivas originarias de la fuerza aplicada en su inserción. Son comunes en pacientes del sexo masculino y actualmente pueden ser consideradas las principales causas notificadas de estenosis uretral ${ }^{(2,4-6)}$.

En la práctica del cateterismo urinario, así como en muchos otros procedimientos practicados por la enfermería, existe dicotomía entre la práctica, la enseñanza y las evidencias científicas sobre el tema ${ }^{(1,6)}$, lo que pone en riesgo a profesionales y pacientes. Le cabe al enfermero practicar, capacitar y supervisar al equipo de enfermería en la introducción y mantenimiento del cateterismo urinario y les compete a los cursos de formación en Enfermería capacitar al graduando, contextualizándole con la realidad de la ciencia, de la constante obligación de re-evaluación de las prácticas y conductas, a través del desarrollo del sentido crítico y de la capacidad de búsqueda por el perfeccionamiento profesional.

En ese contexto, la finalidad de este estudio es verificar cómo el procedimiento de inserción del catéter urinario permanente ha ocurrido en la práctica clínica de enfermería.

\section{MATERIAL Y MÉTODO}

Estudio descriptivo, desarrollado con objeto de describir y documentar los aspectos de la inserción del catéter urinario. Tras la autorización del Comité de Ética en Investigación de la Escuela de Enfermería de Ribeirão Preto - USP (Parecer 0961/2008) y la firma del Término de Consentimiento Libre e Informado, los datos fueron recolectados mediante entrevista en todas las instituciones hospitalarias de un municipio del interior del estado de São Paulo. De los 13 hospitales del municipio, nueve concordaron en participar de la investigación. En cada una de las instituciones participantes, fue entrevistado un enfermero, designado por la propia institución. Todos los enfermeros entrevistados participaron en sus instituciones en el establecimiento y en la implantación del protocolo de cateterismo urinario permanente.

Para la realización de la entrevista se utilizó un formulario semi-estructurado compuesto por informaciones demográficas, de identificación de la institución y del profesional entrevistado, además de datos específicos de los protocolos institucionales destinados a la realización del cateterismo urinario permanente. Para fines de caracterización, las instituciones investigadas fueron descritas en orden alfabético y clasificadas respecto al tamaño como pequeña, media y grande y respecto al régimen de financiación en pública, privada o mixta. Los resultados 
alcanzados en esa fase fueron analizados por revisión de la literatura, con el uso de estadística descriptiva, siendo presentados con figuras y relato discursivo ${ }^{(7)}$.

\section{RESULTADOS}

Entre los 13 hospitales del municipio, 9 concordaron en participar del estudio, 5 $(55,6 \%)$ privados, $3(33,3 \%)$ públicos y $1(11,1 \%)$ filantrópico. Todas las instituciones investigadas poseen el procedimiento de cateterismo urinario estandarizado. El cateterismo urinario se aplica solamente tras prescripción médica y los profesionales responsables por su realización son el enfermero, el equipo enfermero y el equipo médico. Es también la prescripción médica quien determina la suspensión del uso del catéter urinario.

Según la descripción de los entrevistados, los materiales descritos como utilizados para la práctica del procedimiento fueron: (9) bolsa recolectora, (9) guantes estériles, (9) catéter de Foley $(12,14,16)$, (8) povidona yodada (PY) - tópico y desinfectante, (7) cuba redonda, (7) clorhexidina (acuosa, desinfectante), (7) clorhidrato de lidocaína gel $(2 \%)$ en tubo, (7) agua destilada, (6) bolas de algodón, (6) jeringa $20 \mathrm{ml}$, (5) cuba riñón 5, (5) gasa, (4) cinta adhesiva (esparadrapo, microporo), (3) campo quirúrgico, (3) jeringa de $10 \mathrm{ml}$, (2) bandeja, (1) catéter de silicona (UTI), (1) pinzas modelo pean, (1) jeringa con clorhidrato de lidocaína gel (2\%), (1) alcohol $70 \%$. Los cuidados y conductas informados por los entrevistados en la práctica del cateterismo urinario permanente están descritos en la Figura 1.

Figura 1: Cuidados y conductas informados por los entrevistados en la práctica del cateterismo urinario permanente. Ribeirão Preto, 2011.

\begin{tabular}{|c|c|c|c|c|c|c|c|c|c|}
\hline $\begin{array}{l}\text { Hospitales } \\
\text { Cuidados } \\
\text { y conductas }\end{array}$ & A & B & C & D & $E^{*}$ & $F$ & G & $\mathrm{H}$ & I \\
\hline $\begin{array}{l}\text { Higiene de las } \\
\text { Manos }\end{array}$ & $X$ & $X$ & $\mathrm{NI}$ & $X$ & $\mathrm{NI}$ & $X$ & $X$ & $X$ & $X$ \\
\hline $\begin{array}{l}\text { Privacidad del } \\
\text { paciente }\end{array}$ & $X$ & $X$ & $\mathrm{X}$ & $\mathrm{X}$ & $\mathrm{NI}$ & $\mathrm{NI}$ & $\mathrm{NI}$ & $\mathrm{NI}$ & $\mathrm{X}$ \\
\hline $\begin{array}{l}\text { Orientación } \\
\text { Paciente }\end{array}$ & $\mathrm{NI}$ & $\bar{X}$ & $\bar{X}$ & $\mathrm{NI}$ & $\mathrm{NI}$ & $\bar{X}$ & $\bar{X}$ & $\mathrm{NI}$ & $\bar{X}$ \\
\hline $\begin{array}{l}\text { Higiene íntima } \\
\text { del paciente }\end{array}$ & $\mathrm{X}$ & $\mathrm{X}$ & $\mathrm{X}$ & $\bar{X}$ & $\bar{X}$ & $\mathrm{NI}$ & $\bar{X}$ & $\bar{X}$ & $\mathrm{X}$ \\
\hline $\begin{array}{l}\text { Número } \\
\text { de profesionales } \\
\text { involucrados }\end{array}$ & 2 & 102 & 2 & 1 & 1 & 2 & 2 & 2 & 102 \\
\hline $\begin{array}{lr}\text { Cantidad } & \text { de } \\
\text { Clorhidrato } & \text { de } \\
\text { lidocaína } & \text { gel } \\
(2 \%) & \\
& \end{array}$ & $\mathrm{NI}$ & $\begin{array}{l}8 \text { a10 } \\
\mathrm{ml}\end{array}$ & $8 \mathrm{ml}$ & $\begin{array}{l}5 \text { a } 10 \\
\mathrm{ml}\end{array}$ & $\mathrm{NI}$ & 7 a $10 \mathrm{ml}$ & $5 \mathrm{ml}$ & $\mathrm{NI}$ & $8 \mathrm{a} 10 \mathrm{ml}$ \\
\hline Antisepsia & Sí & Sí & Sí & Sí & Sí & Sí & Sí & Sí & Sí \\
\hline $\begin{array}{l}\text { Test balón del } \\
\text { catéter }\end{array}$ & $\begin{array}{l}\text { Agua } \\
\text { destila } \\
\text { da }\end{array}$ & $\begin{array}{l}\text { No } \\
\text { hace }\end{array}$ & Aire & $\begin{array}{l}\text { Agua } \\
\text { destilada }\end{array}$ & Aire & $\begin{array}{l}\text { Agua } \\
\text { destilada }\end{array}$ & Aire & $\begin{array}{l}\text { Agua } \\
\text { destilada }\end{array}$ & $\begin{array}{l}\text { Agua } \\
\text { destilada }\end{array}$ \\
\hline $\begin{array}{l}\text { Tracciona } \\
\text { catéter } \\
\text { fijación }\end{array}$ & Sí & No & Sí & No & Sí & $\mathrm{NI}$ & $\mathrm{NI}$ & Sí & No \\
\hline
\end{tabular}




\begin{tabular}{|c|c|c|c|c|c|c|c|c|c|}
\hline $\begin{array}{lr}\text { Introduce } & \\
\text { catéter } \\
\text { salida de } \\
\text { orina }\end{array}$ & Sí & No & No & $+3 \mathrm{~cm}$ & No & No & No & No & No \\
\hline $\begin{array}{ll}\text { Región } & \text { de } \\
\text { fijación } & \text { del } \\
\text { catéter } & \end{array}$ & $\begin{array}{l}\text { O } \\
\text { hipogá } \\
\text { strica o } \\
\text { flanco } \\
\text { lateral } \\
\\
9 \\
\text { lateral } \\
\text { del } \\
\text { muslo }\end{array}$ & 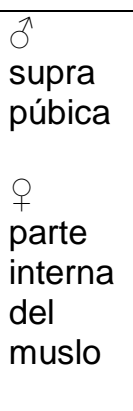 & $\begin{array}{l}\text { Â región } \\
\text { supra } \\
\text { púbica } \\
\text { ótateral } \\
\text { del } \\
\text { muslo }\end{array}$ & $\begin{array}{l}\text { J'región } \\
\text { supra } \\
\text { púbica } \\
\text { qLateral } \\
\text { del } \\
\text { muslo }\end{array}$ & $\begin{array}{l}\text { Plateral } \\
\text { del } \\
\text { muslo }\end{array}$ & $\begin{array}{l}\text { o región } \\
\text { supra } \\
\text { púbica } \\
\text { Plateral del } \\
\text { muslo }\end{array}$ & $\begin{array}{l}\text { A región } \\
\text { supra } \\
\text { púbica } \\
\text { qlateral } \\
\text { del } \\
\text { muslo }\end{array}$ & $\begin{array}{l}\text { đ región } \\
\text { supra } \\
\text { púbica } \\
\text { qlateral } \\
\text { del } \\
\text { muslo }\end{array}$ & $\begin{array}{l}\text { ô región } \\
\text { supra } \\
\text { púbica } \\
\text { +lateral } \\
\text { del } \\
\text { muslo }\end{array}$ \\
\hline $\begin{array}{l}\text { Registro del } \\
\text { procedimiento } \\
\text { en la bolsa } \\
\text { recolectora }\end{array}$ & No & Sí & No & Sí & No & No & No & No & Sí \\
\hline $\begin{array}{l}\text { Registro en el } \\
\text { archivo }\end{array}$ & No & No & Sí & Sí & No & No & No & No & No \\
\hline $\begin{array}{lr}\text { Intervalo } & \text { del } \\
\text { desecho } & \text { del } \\
\text { volumen de } \\
\text { orina de la bolsa } \\
\text { recolectora }\end{array}$ & $\begin{array}{l}\text { Al } \\
\text { término } \\
\text { de } \\
\text { plantón } \\
\text { o en } \\
\text { caso } \\
\text { de } \\
\text { volum } \\
\text { en } \\
\text { superi } \\
\text { or a a } \\
1.200 \\
\text { ml }\end{array}$ & $\begin{array}{l}\text { Volum } \\
\text { en } \geq \text { a } \\
80 \% \\
\text { de la } \\
\text { capaci } \\
\text { dad de } \\
\text { la } \\
\text { bolsa } \\
\text { recolect } \\
\text { ora }\end{array}$ & $\begin{array}{l}\text { Al } \\
\text { término } \\
\text { de turno }\end{array}$ & $\begin{array}{l}\text { De } 4 \text { en } \\
4 \text { horas }\end{array}$ & $\begin{array}{l}\text { Volumen } \\
\geq 600 \mathrm{ml} \\
\text { de orina }\end{array}$ & $\begin{array}{l}\text { Al término } \\
\text { de turno }\end{array}$ & $\begin{array}{l}\text { En las } \\
\text { unidades } \\
\text { de } \\
\text { ingreso } \\
\text { al } \\
\text { término } \\
\text { de } \\
\text { turno. } \\
\text { En la } \\
\text { UTI de } \\
2 / 2 \text { horas }\end{array}$ & $\begin{array}{l}\text { Al } \\
\text { término } \\
\text { de turno }\end{array}$ & $\begin{array}{l}\text { Volumen } \\
\geq \text { a } 80 \% \\
\text { de la la } \\
\text { capacidad } \\
\text { de la } \\
\text { bolsa } \\
\text { recolecto } \\
\text { ra }\end{array}$ \\
\hline $\begin{array}{ll}\text { Frasco/ } & \text { Cáliz } \\
\text { individual para } \\
\text { desecho de la } \\
\text { orina }\end{array}$ & No & Sí & No & Sí & Sí & Sí & Sí & Sí & Sí \\
\hline $\begin{array}{l}\text { Cuidados de } \\
\text { Higiene } \\
\text { paciente y } \\
\text { catéter diarios }\end{array}$ & $\begin{array}{l}\text { Higiene } \\
\text { íntima } \\
\text { diaria } \\
\text { y uso } \\
\text { de } \\
\text { alcohol } \\
70 \% \\
\text { para } \\
\text { limpieza } \\
\text { de la } \\
\text { extremi } \\
\text { dad del } \\
\text { sistema } \\
\text { cada } \\
24 h\end{array}$ & $\begin{array}{l}\text { Alcohol } \\
70 \% \\
\text { para } \\
\text { desinfe } \\
\text { cción } \\
\text { de la } \\
\text { extremi } \\
\text { dad del } \\
\text { sistema } \\
\text { cada } \\
24 \mathrm{~h}\end{array}$ & $\begin{array}{l}\text { Higiene } \\
\text { diaria } \\
\text { del } \\
\text { meato }\end{array}$ & $\begin{array}{l}\text { Higiene } \\
\text { diaria } \\
\text { del } \\
\text { meato }\end{array}$ & $\begin{array}{l}\text { Alcohol } \\
70 \% \\
\text { para } \\
\text { desinfec } \\
\text { ción de } \\
\text { la extremi } \\
\text { dad del } \\
\text { sistema } \\
\text { cada } \\
24 \mathrm{~h}\end{array}$ & $\begin{array}{l}\text { Alcohol } \\
70 \% \text { para } \\
\text { desinfec } \\
\text { ción de la } \\
\text { extremidad } \\
\text { del sistema } \\
\text { cada } 24 \mathrm{~h}\end{array}$ & No & No & $\begin{array}{l}\text { Alcohol } \\
70 \% \\
\text { para } \\
\text { desinfec } \\
\text { ción de } \\
\text { la } \\
\text { extremi } \\
\text { dad del } \\
\text { sistema } \\
\text { cada } \\
24 \mathrm{~h}\end{array}$ \\
\hline
\end{tabular}

$\mathrm{Nl}=$ No informó ${ }^{\star}$ Trátase de un hospital que sólo atiende pacientes del sexo femenino

\section{DISCUSIÓN}

En la práctica clínica del enfermero, los procedimientos son una práctica común, que permea la atención al paciente, forma parte de la formación del profesional y debe merecer inversión de investigación. 
Ante los diversos procedimientos comúnmente practicados, el estudio del cateterismo urinario permite observar que en las publicaciones relacionadas al tema por la enfermería, es pequeño el grupo de temas, y que estos poco se relacionan con el método, la innovación y la incorporación de nuevas tecnologías para su concretización.

En las instituciones investigadas, el procedimiento es practicado de manera ritualista respecto al método y los materiales, lo que pone en riesgo al paciente y también a los profesionales. Se debe subrayar el uso de la construcción de protocolos en las instituciones para mejorar la calidad y seguridad en la utilización de ese procedimiento $^{(8-9)}$.

Respecto a los profesionales, se puede destacar en la muestra medidas relacionadas con la higiene de las manos y el número de recursos humanos. La higiene de las manos es una medida simple y esencial, utilizada para la eliminación de los microorganismos transitorios que residen en la capa superficial de la piel. De bajo coste y de gran valor para la prevención de infecciones hospitalarias, debe ocurrir antes y después de la inserción del catéter urinario o la manipulación del perineo ${ }^{(3,10)}$, lo que no fue evidenciado en ese estudio. Los factores limitantes abarcan la deficiencia de la estructura física y de materiales de las instituciones, la falta de adhesión, conocimiento y exceso de actividades de los profesionales ${ }^{(10-11)}$.

Las cuestiones de dimensionamiento de personal indican la posibilidad de que más de un individuo esté involucrado en la práctica del procedimiento, lo que fue comentado en siete de las instituciones investigadas, y están vinculadas directamente a la calidad de la atención al paciente, disminución de los índices de infección hospitalaria y cuestiones financieras de las organizaciones ${ }^{(11)}$.

Respecto a los pacientes, es función del enfermero garantizar su privacidad en la realización de los procedimientos, evitando la exposición innecesaria y velando por su bienestar. La privacidad es una necesidad y un derecho fundamental para la individualidad del paciente, y no fue mencionada por cuatro sujetos del estudio. Cuando violada, puede causar sentimientos negativos y experiencias angustiantes ${ }^{(3,12,13)}$. Sin embargo, cuando aliada a orientación previa a la inserción del catéter urinario, contribuirá a promover la reducción de la ansiedad, del constreñimiento, el aumento de la confianza y el intercambio de informaciones con el profesional, minimizando riesgos, incomodidad y traumas uretrales. Es derecho del paciente y deber del profesional el intercambio de informaciones sobre los riesgos, costes y beneficios de los procedimientos indicados ${ }^{(14)}$.

La higiene previa a la introducción del catéter urinario no fue mencionada por uno de los sujetos entrevistados. Es una medida importante de prevención de la ITU, ya que evita la entrada de microorganismos en la uretra. Debe ser practicada con agua tibia y jabón, sin provocar traumas, de manera que todas las áreas del perineo estén limpias. Al final de su práctica, demanda la remoción completa del jabón y secado del área ${ }^{(5,15)}$.

Respecto a la utilización de los antisépticos, diferente de los datos mencionados por los sujetos, deben ser empleados solamente en la forma acuosa, debido al contacto con mucosas. No necesitan de mitos y rituales relacionados con la manera como son administrados, aunque deben ser mejor estudiados, ya que todavía no existe consenso sobre la eficacia de su uso ${ }^{(5,16-17)}$. 
El uso del calibre adecuado es fundamental para la no ocurrencia de traumas en la uretra, y el diámetro interno [que corresponde a 1/3 del número del catéter en la escala French (Fr) siempre debe ser elegido de acuerdo con el confort y la seguridad del paciente. Catéteres de menores calibres disminuyen riesgos y la sensibilidad dolorosa del paciente ${ }^{(3,14)}$. Fueron mencionados como utilizados catéteres de látex en los calibres $10 \mathrm{Fr}, 12 \mathrm{Fr}$ y $14 \mathrm{Fr}$ y solamente en una institución: el de silicona para pacientes en terapia intensiva. Aunque el catéter de silicona tiene mayor coste, posee características superiores porque no conduce electricidad y es resistente al agua y a factores oxidantes.

Para minimizar la sensibilidad del paciente en la práctica del procedimiento, es necesario el uso de lubricante o la combinación de lubricante y anestésico. Los primeros lubricantes y anestésicos utilizados en procedimientos uretrales remontan al final del siglo XVIII e inicio del siglo XIX. Fueron producidos a base de aceite, geles acuosos de gomas vegetales que cuando asociados a agentes anestésicos, promovieron gran avance en los procedimientos uretrales ${ }^{(18-20)}$.

Básicamente, la función de los lubrificantes es proteger la mucosa uretral, a través de una excelente lubricación para disminuir la fricción. Son mejor evaluados cuando posibilitan visibilidad óptica, control de flora microbiana, alta conductibilidad eléctrica y efectos anestésicos. En este sentido, pueden ser utilizados solos, con adición de anestésicos (lidocaína 2\%) y antisépticos (clorhexidina). Cuando asociados a la clorhexidina, ayudan en procesos de contaminación, pero normalmente llevan a mayor sensibilidad. Cuando combinados con la lidocaína al $2 \%$, parecen disminuir la sensibilidad de la mucosa, pero existen controversias en la literatura respecto a su efecto anestésico. En esas situaciones, se recomienda además qué cuidados deben ser tomados debido a los efectos adversos de la absorción del anestésico en mucosa no íntegra ${ }^{(18-20)}$.

De forma divergente de los resultados encontrados, a pesar de discusiones entre los autores, se recomienda en el cateterismo urinario el uso de $20 \mathrm{ml}$ de lubricantes asociados a anestésicos durante diez a veinte minutos antes del procedimiento en hombres y niños; para mujeres se recomienda solamente el lubricante.

El test previo del balón está destinado a confirmar su integridad, previniendo traumas y la salida accidental del catéter. Siempre debe ser practicado con la ayuda de una jeringa y agua destilada en el volumen indicado en las instrucciones del fabricante ${ }^{(21)}$. Tras la introducción del catéter, el balón debe ser insuflado con seguridad, o sea, después de visualizar el drenaje de orina, lo que causa controversias respecto a las recomendaciones ${ }^{(3)}$ de uso de sistema cerrado durante la inserción del catéter.

El catéter debe ser fijado con cinta hipoalergénica. La fijación correcta impide que se mueva en la uretra, previniendo tracciones indeseables y/o extracción accidental. En el sexo masculino, debe ser practicada preferencialmente en el abdomen, como forma de prevenir la presión del conjunto sobre el ángulo peniano-escrotal. En el sexo femenino, debe ser fijada en la región antero-lateral o parte interna del muslo ${ }^{(22-23)}$.

En el mantenimiento del catéter permanente, la higiene del perineo se ha revelado como una estrategia eficaz en la disminución de la incidencia de ITU ${ }^{(24-26)}$. Necesita ser practicada diariamente en el meato uretral, con agua y jabón durante el baño. 
Durante el procedimiento, además, debe ser movilizado el catéter, garantizando la limpieza de todas las áreas y la prevención del trauma de tejido ${ }^{(3,5,16)}$.

Con objeto de minimizar los riesgos de infección urinaria, los sistemas cerrados de drenaje de orina son los recomendados. Utilizan bolsas plásticas y desechables que deben ser mantenidas inferior a la vejiga y sin tocar el suelo. La orina debe ser desechada respetándose la clínica del paciente, en intervalos periódicos y recipientes individuales, manteniéndose las medidas de higiene y de precauciones estándar para la integridad del paciente y del profesional.

El registro de la actividad muestra la calidad del trabajo del enfermero y permite el rastreo de informaciones. Debe contener datos temporales, de los profesionales involucrados, motivos de la cateterización, materiales utilizados, procedimientos practicados, respuesta del paciente y posibles problemas. Puede ser reforzado con el registro de las informaciones en la bolsa recolectora ${ }^{(3,14)}$.

\section{CONCLUSIÓN}

El cateterismo urinario permanente es un procedimiento complejo que involucra la gestión de materiales, equipos, personas y procesos, además de acciones vinculadas al respecto y la seguridad del paciente, procedimiento y documentación, lo que tiene una relación muy estrecha con las instituciones, sus procesos de capacitación, protocolos y financiación.

En las instituciones investigadas, el procedimiento ha sido practicado de manera distinta, no uniforme, sin seguridad y sin fidelidad al método. Considerando que este procedimiento es desarrollado de manera rutinaria en la atención al paciente, necesita de mayor compromiso de los profesionales respecto a los derechos del paciente y la precisión técnica, además de mayor inversión en investigaciones para aclaración de los puntos que todavía no pueden ser comprobados.

\section{REFERENCIAS}

1- Lenz LL: Cateterismo vesical: cuidados, complicações e medidas preventivas. ACM Arq Catarin Med. 2006; 35:82-91.

2- Mazzo A, Godoy S, Alves LM, Mendes IAC, Trevizan MA, Rangel EML: Cateterismo urinário: facilidades e dificuldades relacionadas à sua padronização. Texto \& Contexto Enferm. 2011; 20:333-9.

3- Center for Disease Control and Prevention (CDC): Guideline for prevention of catheter-associated urinary tract infections. Atlanta, EUA. 2009.

4- Canales BK, Weiland D, Reardon S, Monga M: Urethral catheter insertion forces: a comparison of experience and training. Int Braz J Urol. 2009; 35:84-9.

5- Mundy AR, Andrich DE: Urethral trauma. Part I: Introduction, history, anatomy, pathology, assessment and emergency management. BJU Int. 2011; 310-27.

6- Mazzo A, Gaspar AACS, Mendes IAC, Trevizan MA, Godoy S, Martins JCA: Cateter urinário: mitos e rituais presentes no preparo do paciente. Acta Paul Enferm. 2012; 25:889-94.

7- Polit DF, Beck CT, Hungler BP: Fundamentos de Pesquisa em Enfermagem. Métodos, avaliação e utilização. 2004, Porto Alegre, Artmed.

8- Lima LS, Araújo EC,Bezerra SMMS, Linhares FM, Lima AKA. Infecciones del tracto urinario en pacientes con sonda vesical de demora internados en una Unidad de Vigilancia Intensiva en Recife (PE), Brasil. Enfermería Global, 2007; 6(2): 1-11. 
9- Torres Alaminos, $\mathrm{M}^{\mathrm{a}}$ Angustias. Medidas preventivas para evitar complicaciones de salud derivadas del uso de sondajes vesicales en pacientes lesionados medulares. Enfermería Global, 2013; 12(30): 370-378.

10- Neves ZCP, Tipple AFV, Souza ACS, Pereira MS, Melo DS, Ferreira LR: Hand hygiene: the impact of incentive strategies on adherence among healthcare workers from a newborn intensive care unit. Rev Latino-Am Enfermagem. 2006; 14:546-52.

11- Oliveira AC, Cardoso CS, Mascarenhas D: Precauções de contato em Unidade de Terapia Intensiva: fatores facilitadores e dificultadores para adesão dos profissionais. Rev Esc Enferm. USP. 2010; 44:161-65.

12- Magalhães AMM, Riboldi CO, Dall'Agnol CM: Planejamento de recursos humanos de enfermagem: desafio para as lideranças. Rev Bras Enferm. 2009; 62:608-12.

13- Pupulim JSL, Sawada NO: O cuidado de enfermagem e a invasão da privacidade do doente: uma questão ético-moral. Rev Latino-Am Enfermagem. 2002; 10:433-438.

14- Gasparino RC, Guirardello EB: Sentimento de invasão do espaço territorial e pessoal do paciente. Rev Bras Enferm. 2006; 59:652-55.

15- Geng V, Emblem EL, Gratzel S, Incesu O, Jensen K: European Associattion of Urology Nurses. Good practices in health care. Uretheral catheterization. Section 2. Male, female and paediatric intermittent catheterization, 2006.

16- Leaver RB: The evidence for urethral meatal cleansing. Nurs Stand. 2007; 21:394.

17- Webster J, Hood RH, Burridge CA, Doidge ML, Phillips KM, George N: Water or antiseptic for periurethral cleaning before urinary catheterization: a randomized controlled trial. Am J Infect Control. 2001; 29:389-94.

18- Al-Farsi S, Oliva M, Davidson R, Richardson SE, Ratnaplan S. Peri-uretheral cleaning prior to urinary catheterization in children: sterile water versus $10 \%$ povidineiodine. Clin Pediatr. 2009; 48:656-60.

19- Tzortzis V, Gravas S, Melekos MM, Rosette, JJ: Intraurethral Lubricants: a critical literature review and recommendations. J Endourol. 2009; 23:821-6.

20- Aaronson DS, Walsh TJ, Smith JF, Davies BJ, Hsieh MH, Konety BR: Metaanalysis: does lidocaine gel before flexible cystoscopy provide pain relief? BJU International. 2009; 104:506-10.

21- Gordetsky J, Bendana E, O'Brien J, Rabinowiz R. (Almost): Painless surgery: A historial review of the evolution of intraurethral anesthesia in urology. Urology. 2011; 77:236-7.

22- Perry AG, Potter PA. Guia completo de procedimentos e competência de enfermagem. Rio de Janeiro, Elsevier. 2012; p. 640.

23- Napoleão AA, Caldato VG, Petrilli Filho JF. Diagnóstico de enfermagem para o planejamento da alta de homens prostatectomizados: um estudo preliminar. Rev Eletrônica Enferm. 2009; 11: 286-94.

24- Camara F. R: Cateterismo vesical: enfoque urológico. Departamento de Urologia da FMB/UNESP, 2009.2 Disponible en: http://www.urologiabotucatu.com.br/cateterismo.asp

25- Oliveira R, Azevedo N, Cruz I, Andrade M, Santo F.: Urinary tract infection: searching evidence for nursing care. Online Braz J Nurs. 2008; 7.

26- Tsuchida T, Makimoto K, Ohsako S, Fujino M, Kaneda M, Miyazaki T, et al.: Relationship between catheter care and catheter-associated urinary tract infection at Japanese general hospitals: a prospective observation study. Int J Nurs Stud. 2008; 45:352-61.

Recibido: 19 de noviembre 2013; Aceito: 22 de diciembre 2013 
๑ COPYRIGHT Servicio de Publicaciones - Universidad de Murcia 